\title{
Ethylene Glycol Induced Hyperoxaluria Increases Circulating Endothelial Cells in Peripheral Blood: A Possible Link of Urolithiasis to Cardiovascular Diseases
}

\author{
Hakan Sarı ${ }^{1}$, Faruk Yencilek ${ }^{2}$, Gülderen Yanıkkaya Demirel ${ }^{3}$ and Hasan Aydın* \\ ${ }^{1}$ Department of Internal Medicine, Bagcilar Education and Research Hospital, Turkey \\ ${ }^{2}$ Department of Urology, Yeditepe University, Turkey \\ ${ }^{3}$ Department of Immunology, Yeditepe University, Turkey \\ ${ }^{4}$ Department of Endocrinology and Metabolism, Yeditepe University, Turkey
}

*Corresponding author: Hasan Aydın, Department of Endocrinology and Metabolism, Yeditepe University Medical Faculty, Istanbul, Turkey

Submission: 㘹 May 29, 2018; Published: 眥July, 14, 2018

\begin{abstract}
:
The aim of this study was to establish the role of hyperoxaluria on endothelial dysfunction by using Circulating Endothelial Cells (CECs). A total of 24 Sprague Dowley rats have been included into study. Group IA, B and C ( $\mathrm{n}=6$ in each): Hyperoxaluria-induced group; Group II ( $\mathrm{n}=6)$ : Control group. Rats were euthanized after collection of 24-hour urine samples for the analysis of oxalate excretion either at $24^{\text {th }}$ hour (group A), $14^{\text {th }}$ day (group B) or $28^{\text {th }}$ day (group C) and blood samples were collected for the analysis of CECs. Rats in control group were euthanized after collecting 24-hour urine samples. CECs were determined via multicolor flow cytometric method with the help of rat anti-CD 146-PE antibodies together with CD31, CD45 and CD34 monoclonal antibodies. Compared to controls, the amount of CECs was found to be higher in hyperoxaluric group. Highest levels were achieved at day 14 $(1.60 \pm 0.27,1.93 \pm 0.42$, and $1.20 \pm 0.20,24$-hour, 14 -day and 28-day samples, respectively). The amount of CECs correlated positively with urinary oxalate excretion ( $\mathrm{p}=0.002, \mathrm{r}=0.679$ ). Ethylene glycol-induced hyperoxaluria causes systemic endothelial dysfunction. This may contribute to the pathogenesis of increased risk of cardiovascular diseases in urolithiasis.
\end{abstract}

Keywords: Hyperoxaluria; Endothelial dysfunction; Circulating endothelial cells; Urolithiasis; Cardiovascular risk

Abbreviations: CECs: Circulating Endothelial Cells; ADMA: Asymmetrical Di Methyl Arginine; EDTA: Ethylene Diaminic Tetra acetic Acid

\section{Introduction}

Urolithiasis is a common disease with an increasing incidence and prevalence worldwide. Exact pathogenesis of the disease is not clear. Many hypotheses were proposed which some of them included physical factors while others of cellular factors. Although it is a systemic disease, for many years, it was thought to be a disease restricted to kidneys. But recently, it is realized that urolithiasis is closely related with some systemic disorders such as obesity, type 2 diabetes mellitus, hyperlipidemia, hypertension, metabolic syndrome which are very well known risk factors for the development of cardiovascular diseases [1-6].

Endothelial tissue is one of the main targets in atherosclerosis. Several studies have clearly established the key role of endothelial dysfunction on the development of atherosclerosis and it is accepted as one of the earliest predictor of cardiovascular risk $[7,8]$. Endothelial cells are in close relation with renal tubular epithelial cells and interact with each other [9]. Some recent studies reported a local relationship of endothelial nitric oxide synthase in experimentally induced hyperoxaluric animals [10]. But, in one of our recent studies, we demonstrated that hyperoxaluria induces both local and systemic endothelial dysfunction as shown by increased plasma Asymmetrical Di Methyl Arginine (ADMA) levels which is a measure of endothelial dysfunction [11].

There are some other ways of documenting endothelial dysfunction. One of them is to count CECs [12]. Studies have shown a relationship between these cells types and plasma and physiological indicators of vascular damage, and atherosclerotic risk factors [13]. Quantification of CECs is accepted as an important 
marker of cardiovascular disease risk [14]. The aim of this study to establish endothelial dysfunction in hyperoxaluria by using a different method of studying endothelial function other than measuring ADMA, namely Circulating Endothelial Cells (CECs).

\section{Materials and Methods}

A total of 24 Sprague-Dawley rats weighing 200 to 250 gram were included in the study, which was done in accordance with accepted standards of human animal care and use, as deemed appropriate by the Yeditepe University Medical Faculty animal care and use committee. Rats were housed in a specific pathogenfree room with an alternating 12 hours of light and darkness at a constant mean \pm SD temperature of $230 \mathrm{C} \pm 10 \mathrm{C}$ and $55 \% \pm 5 \%$ humidity. All rats had free access to distilled water and were fed 15 gram per day per rat of diet, which is the minimum average food intake of rats during acclimatization. All animals were screened for possible urinary tract and parasitic infections by urinalysis and microscopic examination of urine samples before collection of 24 hour urine specimens, which may ultimately alter the presence and degree of hyperoxaluria induced renal parenchymal alterations during study procedures. No rat had any evidence of infection and no specific treatment was needed.

The animals were then divided into 2 groups. Group 1 with induced hyperoxaluria included 18 rats and further divided into 3 subgroups of 6 in each (group 1A-early phase 24-hour period, group 1B-14-day period and group 1C-long-term 28-day period) was given a hyperoxaluria inducing diet of $0.75 \%$ ethylene glycol in distilled drinking water for 2 weeks which is the accepted time period to induce hyperoxaluria in rats. Group 1C was selected both to observe long term effects and changes after exposure to ethylene glycol stops. Group $2(\mathrm{n}=6)$ was control group. No specific medication was given. Animals were sacrificed after collection of 24-hour urine for the detection of urinary oxalate excretion rate. Animals in group $1 \mathrm{~A}$ were sacrificed 24 hour after exposure to ethylene glycol, 14 days in group $1 \mathrm{~B}$ and 28 days in group $1 \mathrm{C}$ and blood samples were obtained. Rats in control group sacrificed after collecting 24-hour urine samples. Blood samples in control group also obtained for CECs. Since there was no intervention with any medicine and chemicals, they were not followed for 28 days.

\section{Detection of circulating endothelial cells}

Blood samples were collected into Ethylene Diaminic Tetra aceticAcid (EDTA) containing purple top collection tubes. In order to be as non-traumatic as possible, blood samples were collected with cardiac puncture. Blood counts for white blood cells were completed by cell count in Neubauer chambers. Circulating endothelial cells were determined via multicolor flow cytometric method with the help of rat anti-CD 146-PE antibodies (Stem Cell Technologies, Canada) together with CD31, CD45 and CD34 monoclonal antibodies (BioLegend and Beckman Coulter. USA, respectively). CD45-specific antibody was used for immunostaining of hematopoietic cells, while human CD31 antibodies were used for immunostaining of endothelial cells as previously described [15]. Five microliters of monoclonal antibodies were mixed and pipetted onto 100 microliters of blood in each sample tube. After fifteen minutes of incubation at room temperature in dark, tubes were washed twice to eliminate the excess amount of antibodies. Then samples were suspended in buffer solution for immune phenol typing (PBS-Phosphate Buffer solution containing 2\%BSABovine serum albumin). Then cells were analyzed on a five color FC500 cytometer (Beckman Coulter, USA) which has been daily checked for laser alignment (Flow Check. Beckman Coulter, USA) and fluorescence calibration (Flow Set, Beckman Coulter, USA). CD45negCD31+CD34+CD146+ cells were evaluated as CECs [16], CD45+CD146+ cells were considered as $\mathrm{T}$ cell subset (data not shown). A non-stained sample was used as negative control for each sample. The cell count was adjusted to count $1 \times 106$ cells per sample. The samples which did not reach to these numbers were left out of the study. List mode data analysis was completed with CXP II software (Beckman Coulter, USA).

\section{Statistical Analysis}

Statistical data were analyzed by SPSS $₫ 15.0$ for Windows $₫$. Data were subjected to normality test (Kolmogorov Smirnov test) and determined to be normally distributed. Parametric tests were used for the analysis. Descriptive statistics were presented as mean \pm SD. Data for the change in each parameter during study period were analyzed with unpaired t test. Pearson test was used for the correlation analysis. Two-tailed $p$ values less than 0.05 were taken as significant.

\section{Results}

\section{The amount of circulating endothelial cells}

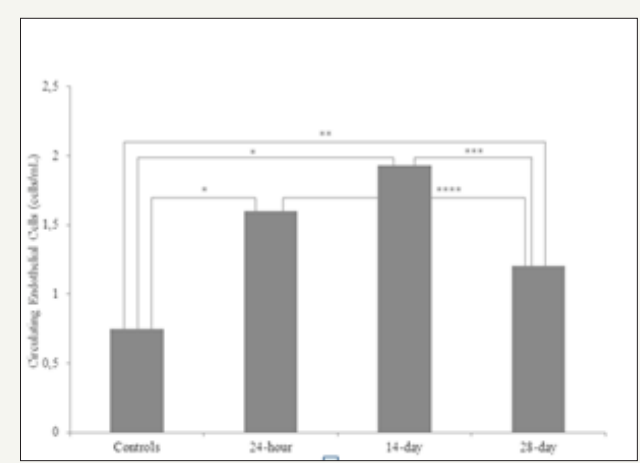

Figure 1: Circulating CD146+ cell population in EG treated rats and controls $\left({ }^{*} \mathrm{p}<0.0001,{ }^{* *} \mathrm{p}=0.002,{ }^{* * *} \mathrm{p}=0.003,{ }^{* * * *} \mathrm{p}=0.01\right)$. 
Compared to controls, the amount of CECs was found to be higher in hyperoxaluric group (Figure 1). Circulating endothelial cells start to increase in the circulation immediately after hyperoxaluric state. First increase was observed at 24-hour period (1.60 \pm 0.27 cells $/ \mathrm{ml}, \mathrm{p}<0.0001)$. The amount of CECs remained high at 14 -day samples $(1.93 \pm 0.42$ cells $/ \mathrm{ml}, \mathrm{p}<0.0001)$ and continued throughout the study period 28 -day $(1.20 \pm 0.20$ cells $/ \mathrm{ml}, \mathrm{p}=0.002)$. Highest level was observed at day 14.Twenty four hour samples did not differ statistically from 14-day samples but significantly higher than 28-day samples ( $\mathrm{p}=0.168$ and 0.01 , respectively). The amount of CECs decreased through the end of the study and amount of cells at day 28 was significantly lower than the 24-hour and 14-day samples ( $\mathrm{p}=0.01$, and $\mathrm{p}=0.003$, respectively) (Figure 1$)$.

\section{The amount of urinary oxalate excretion}

Hyperoxaluria was confirmed by 24-hour urinary excretion which was higher than control group throughout the study protocol (40.2 $\pm 12.9,253.5 \pm 133.4,781.2 \pm 100.2,383.5 \pm 93.7 \mathrm{mg}$ oxalate/gr creatinine/day, for controls, 24-hour, 14-day and 28-day samples, respectively). Exposure to ethylene glycol resulted in higher urinary oxalate levels immediately and firstly observed at $24^{\text {th }}$ hours ( $p<0.0001,24$-hour samples vs. controls) but highest level was observed at 14 th day ( $\mathrm{p}<0.0001,24$-hour samples vs. 14 -day and 14-day vs. 28-day) and its level steadily decreased throughout the end of the study period but was still higher than control group $(\mathrm{p}<0.0001)$. Oxalate excretion rate at 28-day samples was similar to 24-hour samples ( $\mathrm{p}=0.309$ ) (Figure 2).

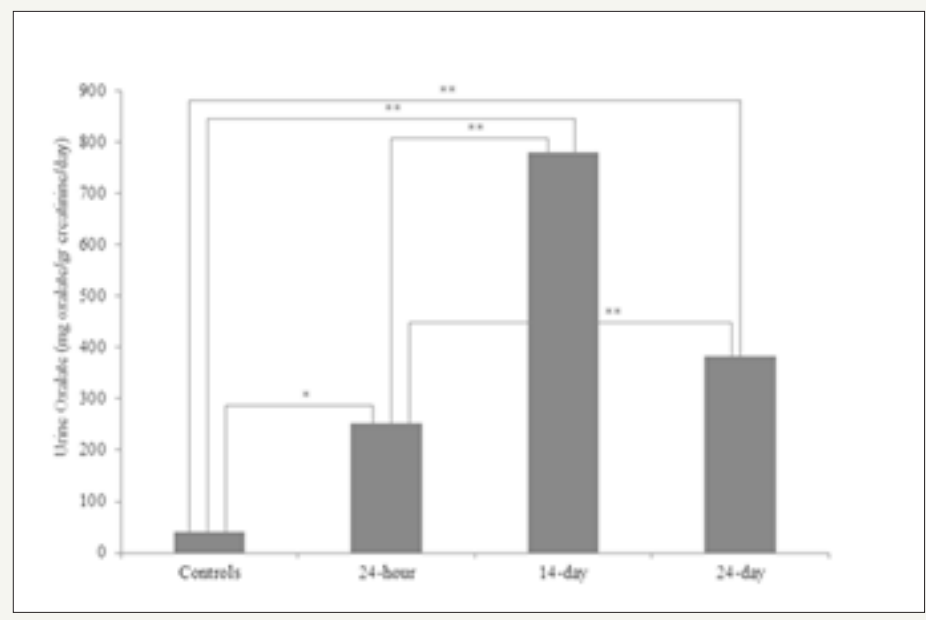

Figure 2: Urinary oxalate excretion rate in EG treated rats and controls $\left({ }^{*} \mathrm{p}=0.003,{ }^{* *} \mathrm{p}<0.001\right)$.

\section{Correlation analysis}

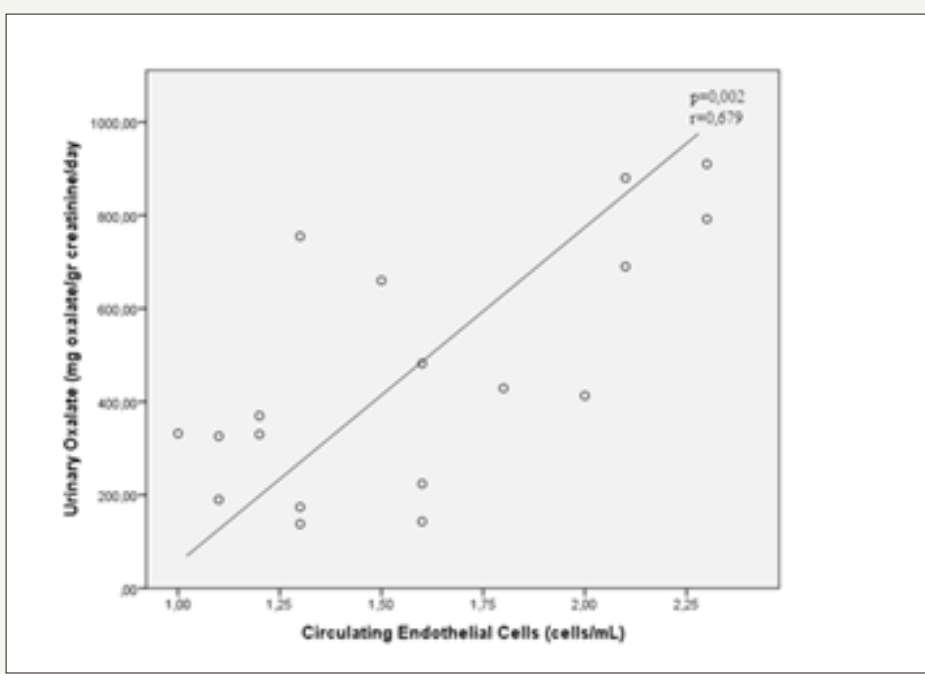

Figure 3: Correlation between urine oxalate excretion and CECs. As urinary oxalate excretion increases circulating endothelial cells increase.

Urinary oxalate excretion rate was positively correlated with the amount of circulating endothelial cells $(p=0.002, r=0.679)$ (Figure 3).

\section{Discussion}

Our results, for the first time demonstrated that circulating endothelial cells increases in hyperoxaluric state. This may help to 
explain the pathogenesis of increased cardiovascular disease risk in urolithiasis.Both in epidemiological and clinical aspects, there are many similarities between urolithiasis and metabolic syndrome. The prevalence of urolithiasis and metabolic syndrome increased over last decades. Every single component that constitute metabolic syndrome that is central obesity, diabetes mellitus, hypertension and dyslipidemia both by its own or together have a direct or indirect relationship with urolithiasis. It is well known that the prevalence of urolithiasis is more common in obese people. As body mass index increases, the risk of urolithiasis increases nearly 2-fold [17]. Urinary crystal excretion also increases with increased body weight. There are studies that show an increased risk of urolithiasis in patients with hypertension, hyperlipidemia and type 2 diabetes mellitus. Besides, patients with urolithiasis also have increased prevalence of components of metabolic syndrome $[17,18]$.

Cardiovascular disease risk is increased in urolithiasis. Some of the components of well- known cardiovascular disease have been associated with the occurrence of nephrolithiasis in epidemiological manner or with biochemical abnormalities that in turn are related to cardiovascular diseases $[6,19]$. In one of the largest population based studies, 23,349 people were screened in IV Portuguese National Health Survey for documenting possible associations between nephrolithiasis and cardiovascular diseases. After adjusting for age and body mass index, kidney stone formers have higher prevalence of myocardial infarction and stroke compared with non-stone formers [20].

In one of our previous studies, we have demonstrated cardiovascular risk elevation also in patients with urolithiasis such that patients with urolithiasis had increased 10-year cardiovascular disease and mortality risk scores compared to healthy people. The Framingham and SCORE risk score were significantly correlated with urinary calcium and oxalate excretion [6]. A metaanalysis dealt with 6 cohort studies including 49597 patients with kidney stones demonstrated $29 \%$ and $31 \%$ increase in myocardial infarction and coronary revascularization risk, respectively [21]. In a Canadian cohort study based on universal health care system, 3,195,452 people aged $\geq 18$ years were searched for the development of urolithiasis and cardiovascular events. Compared with people without kidney stones, people who had at least one kidney stone had a higher risk of subsequent acute myocardial infarction; percutaneous transluminal coronary angioplasty/coronary artery bypass grafting and stroke [22].

The exact mechanism of increase in cardiovascular disease development in patients with urolithiasis is not known yet. But, potential factors include metabolic, hormonal or inflammatory responses associated with cardiovascular diseases which may promote a stone forming milieu in urine, environmental factors such as diet and oxidative stress. Studies mainly focused on oxidative stress, inflammation, insulin resistance and endothelial function. Oxidative stress is thought to be the main initiator of both disease states. Reactive oxygen species had been shown to be produced during interaction between $\mathrm{CaOx} /$ calcium phosphate (CaP) crystals and renal epithelial cells in tissue culture and animal model studies [23].
The link between urolithiasis and endothelium is interesting. Endothelial tissue is the inner layer of the vasculature and chemical and physical changes in blood directly affects its function. Since urolithiasis is a systemic metabolic disorder, the idea of dysfunction in endothelium merits consideration. First study on this relationship was carried out by Aydin et al. [11] they have shown that asymmetrical dimethyl arginine increased early in the hyperoxaluric mileu both in local renal tissue and systemic circulation. Linas and Repine had shown that renal proximal epithelial cell transport was controlled by nearby endothelial tissue [9]. Dysfunction in endothelium through oxidative stress induced by hyperoxaluria was thought to be the main driving force. Direct role of endothelial cell dysfunction on renal stone development was the subject of the study done by Sarica et al. [24]. In their cell culture study, human umbilical vein endothelial cells exposed to oxalate had been shown to accelerate oxalate-induced apoptosis of human renal proximal tubule epithelial cells in co-culture system and were prevented by pyrrolidine dithiocarbamate which is a preservative for endothelial cell function.

Several studies have shown that endothelial dysfunction is an early key element in cellular basis of the development of atherosclerosis and is one of the best known predictors of cardiovascular disease risk [8]. There are several ways of documenting endothelial dysfunction. It can be via biochemical markers such as asymmetrical dimethyl arginine, cytokines and growth factors or non-invasively by flow mediated dilatation of forearm vessels [25,26]. Circulating endothelial cells are also known as a novel marker of endothelial damageand increased numbers of circulating endothelial cells had been documented in cardiovascular disease and its risk factors, such as unstable angina, acute myocardial infarction, stroke, diabetes mellitus $[27,28]$.

In this study, for the first time we have shown that circulating endothelial cells increased with the induction of hyperoxaluria. This shows that hyperoxaluria is toxic to the vascular endothelium. The effect was in parallel to urinary oxalate excretion. Maximum number of CECs was observed at maximal urinary oxalate levels. After ethylene glycol administration stopped, urinary oxalate excretion levels dropped but still above the levels of controls. The amount of CECs changed in a similar manner and increases and decreases in levels followed urinary oxalate excretion rate. Positive correlation of CECs with urinary oxalate excretion strengthens the findings. Since hyperoxaluria is a steady and continuous state in calcium oxalate stone disease the effect on endothelium should be continuous in real life conditions.

The effect of hyperoxaluria on CECs was constant throughout the study period. The level did not differ between sampling intervals. Hyperoxaluria had an immediate effect on CECs with a sharp rise within 24 hours indicating an acute toxicity. The stable deterioration of CECs with the level of hyperoxaluria further indicates that once endothelium is injured by oxalate, the dysfunction is continuous. There are certain limitations of our study. First of all, this is an animal study and not always can be projected to humans. Secondly, it would be better to measure metabolic parameters such as insulin resistance and lipid profile or the cytokines such as TNF-alpha, 
hsCRP, IL- 6 that affect endothelial function. Thirdly, plasma oxalate levels could be measured. But, since $90 \%$ of the oxalate is excreted in urine, urinary excretion can be accepted as a good reflection of plasma levels. Fourth, a fifth group representing another animal model of hyperoxaluria since another metabolite of ethylene glycol, other than oxalate, could potentially cause the outcome that is being reported. But in our previous cell culture study, we observed that oxalate by itself induced endothelial dysfunction detected with the measurement of ADMA [24].

\section{Conclusion}

This is the first study demonstrating increased CECs in hyperoxaluric states indicating increased cardiovascular risk in urolithiasis might be related to endothelial dysfunction. This should further be proved in human studies.

\section{References}

1. Taylor EN, Stampfer MJ, Curhan GC (2005) Obesity, weight gain, and the risk of kidney stones. JAMA 293(4): 455-462.

2. Lieske JC, de la Vega LS, Gettman MT, Slezak JM, Bergstralh EJ, et al. (2006) Diabetes mellitus and the risk of urinary tract stones: a populationbased case-control study. Am J Kidney Dis 48(6): 897-904.

3. Masterson JH, Woo JR, Chang DC, Chi T, Esperance JO, et al. (2015) Dyslipidemia is associated with an increased risk of nephrolithiasis. Urolithiasis 43(1): 49-53

4. Cappuccio FP, Siani A, Barba G, Mellone MC, Russo L, et al. (1999) A prospective study of hypertension and the incidence of kidney stones in men. J Hypertens 17(7): 1017-1022.

5. Wong YV, Cook B, Somani BK (2015) The association of metabolic syndrome and urolithiasis. International Journal of Endocrinology, pp.9.

6. Aydin H, Yencilek F, Erihan IB, Okan B, Sarica K (2011) Increased 10year cardiovascular disease and mortality risk scores in asymptomatic patients with calcium oxalate urolithiasis. Urol Res 39(6): 451-458.

7. DiCorleto PE, Chisolm GM 3rd (1986) Participation of the endothelium in the development of the atherosclerotic plaque. Progress in Lipid Research 25(1-4): 365-374.

8. Celermajer DS, Sorensen KE, Bull C, Robinson J, Deanfield JE (1994) Endothelium-dependent dilation in the systemic arteries of asymptomatic subjects relates to coronary risk factors and their interaction. J Am Coll Cardiol 24(6): 1468-1474.

9. Linas SL, Repine JE (1999) Endothelial cells regulate proximal tubule epithelial cell sodium transport. Kidney Int 55(4): 1251-1258.

10. Pragasam V, Sakthivel R, Kalaiselvi P, Rajesh NG, Varalakshmi P (2005) Detection of endothelial nitric oxide synthase and NADPH-diaphorase in experimentally induced hyperoxaluric animals. Urological Research 33(4): 301-308.

11. Aydin H, Yencilek F, Mutlu N, Comunoğlu N, Koyuncu HH, et al. (2010) Ethylene glycol induced hyperoxaluria increases plasma and renal tissue asymmetrical dimethylarginine in rats: a new pathogenetic link in hyperoxaluria induced disorders. J Urol 183(2): 759-764.
12. Erdbruegger U, Haubitz M, Woywodt A (2006) Circulating endothelial cells: A novel marker of endothelial damage. Clin Chim Acta 373(1-2): $17-26$

13. Blann AD, Pretoius A (2006) Circulating endothelial cells and endothelial progenitor cells: Two sides of the same coin, or two different coins? Atherosclerosis 188(1): 12-8.

14. Boos CJ, Lip GY, Blann AD (2006) Circulating Endothelial Cells in Cardiovascular Disease. J Am Coll Cardiol 48(8): 1538-1547.

15. Duda DG, Cohen KS, di Tomaso E, Au P, Klein RJ, et al. (2006) Differential CD146 Expression on Circulating Versus Tissue Endothelial Cells in Rectal Cancer Patients: Implications for Circulating Endothelial and Progenitor Cells As Biomarkers for Antiangiogenic Therapy. J Clin Oncol 24(9): 1449-1453.

16. Tefekli A, Kurtoglu H, Tepeler K, Karadag MA, Kandirali E, et al. (2008) Does the metabolic syndrome or its components affect the outcome of percutaneous nephrolithotomy? J Endourol 22(1): 35-40.

17. Romero V, Akpinar H, Assimos DG (2010) Kidney Stones: A Global Picture of Prevalence, Incidence, and Associated Risk Factors. Rev Urol 12(2-3): e86-96.

18. Gillen DL, Coe FL, Worcester EM (2005) Nephrolithiasis and increased blood pressure among females with high body mass index. Am J Kidney Dis 46(2): 263-269.

19. Hamano S, Nakatsu H, Suzuki N, Tomioka S, Tanaka M, et al. (2005) Kidney stone disease and risk factors for coronary heart disease. Int J Urol 12(10): 859-863.

20. Domingos F, Serra A (2011) Nephrolithiasis is associated with an increased prevalence of cardiovascular disease. Nephrol Dial Transplant 26(3): 864-868.

21. Liu Y, Li S, Zeng Z, Wang J, Xie L, et al. (2014) Kidney stones and cardiovascular risk: a meta-analysis of cohort studies. Am J Kidney Dis 64(3): 402-410.

22. Alexander RT, Hemmelgarn BR, Wiebe N, Bello A, Samuel S, et al. (2014) Kidney stones and cardiovascular events: a cohort study. Clin J Am Soc Nephrol 9(3): 506-512.

23. Khan SR (2012) Is oxidative stress, a link between nephrolithiasis and obesity, hypertension, diabetes, chronic kidney disease, metabolic syndrome? Urol Res 40(2): 95-112.

24. Sarıca K, Aydin H, Yencilek F, Telci D, Yilmaz B (2012) Human umbilical vein endothelial cells accelerate oxalate-induced apoptosis of human renal proximal tubule epithelial cells in co-culture system which is prevented by pyrrolidine dithiocarbamate. Urol Res 40(5): 461-466.

25. Lefer AM, Ma XL (1993) Cytokines and growth factors in endothelial dysfunction. Crit Care Med 21(2 Suppl): S9-14.

26. Celermajer DC, Sorensen KE, Gooch VM, Spiegelhalter DJ, Miller OI, et al. (1992) Non-invasive detection of endothelial dysfunction in children and adults at risk of atherosclerosis. Lancet 340(8828): 1111-1115.

27. Wang C, Li H, Fu P, Zhang S, Xiu R (2005) Serum C-reactive protein and circulating endothelial cells in patients with acute myocardial infarction. Clin Hemorheol Microcirc 32(4): 287-296.

28. McClung JA, Naseer N, Saleem M, Rossi GP, Weiss MB, et al. (2005) Circulating endothelial cells are elevated in patients with type 2 diabetes mellitus independently of HbA(1)c. Diabetologia 48(2): 345-350. 
Creative Commons Attribution 4.0

International License

For possible submissions Click Here

Submit Article

\begin{tabular}{|c|c|c|}
\hline ETUN & 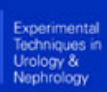 & $\begin{array}{c}\text { Experimental Techniques in Urology \& Nephrology } \\
\text { Benefits of Publishing with us }\end{array}$ \\
\hline & & $\begin{array}{l}\text { - High-level peer review and editorial services } \\
\text { - Freely accessible online immediately upon publication } \\
\text { - Authors retain the copyright to their work } \\
\text { - Licensing it under a Creative Commons license } \\
\text { - Visibility through different online platforms }\end{array}$ \\
\hline
\end{tabular}

